\title{
Rectal 1\% Tenofovir Gel Use Associates with Altered Epidermal Protein Expression
}

\author{
Laura Romas, ${ }^{1,2}$ Kenzie Birse, ${ }^{1,2}$ Kenneth H. Mayer, Max Abou, Garrett Westmacott,, Rebecca Giguere, \\ Irma Febo, Ross D. Cranston, Alex Carballo-Diéguez, Ian McGowan, and Adam Burgener ${ }^{1,2,8}$
}

\begin{abstract}
Rectal use of a $1 \%$ tenofovir (TFV) gel is currently being evaluated for HIV prevention. While careful assessment of mucosal safety of candidate microbicides is a primary concern, tools to assess mucosal toxicity are limited. Mass spectrometry-based proteomics is a sensitive and high-throughput technique that can provide in-depth information on inflammation processes in biological systems. In this study, we utilized a proteomics approach to characterize mucosal responses in study participants involved in a phase 1 clinical trial of a rectal TFV-based gel. Project Gel was a phase 1 randomized (1:1), double-blind, multisite, placebo-controlled trial in which 24 participants received rectal TFV or a universal placebo [hydroxyethyl cellulose (HEC)] over a course of 8 daily doses. Rectal mucosal swabs were collected after 0,1 , and 8 doses and were analyzed by label-free tandem mass spectrometry. Differential protein expression was evaluated using a combination of paired (time-effects) and unpaired (across study arm) $t$-tests, and multivariate [least absolute shrinkage and selection operator (LASSO)] modeling. Within the TFV arm, 7\% $(17 / 249, p<.05)$ and $10 \%(25 / 249, p<.05)$ of total proteins changed after 1 and 8 daily applications of TFV gel, respectively, compared to $3 \%(7 / 249, p<.05)$ and $6 \%(16 / 249, p<.05)$ in the HEC arm. Biofunctional analysis associated TFV use with a decrease in epidermal barrier proteins (adj. $p=1.21 \times 10^{-10}$ ). Multivariate modeling identified 13 proteins that confidently separated TFV gel users (100\% calibration and 96\% crossvalidation accuracy), including the epithelial integrity factors (FLMNB, CRNN, CALM), serpins (SPB13, SPB5), and cytoskeletal proteins (VILI, VIME, WRD1). This study suggested that daily rectal applications of a $1 \%$ TFV gel may be associated with mucosal proteome changes involving epidermal development. Further assessment of more extended use of TFV-gel is recommended to validate these initial associations.
\end{abstract}

Keywords: microbicides, mucosal immunology, systems biology, proteomics, rectal, innate immunity

\section{Introduction}

M ICROBICIDES ARE A TOPICAL prevention strategy against mucosal transmission of HIV. The CAPRISA-004 trial demonstrated that a $1 \%$ tenofovir (TFV) gel was partially effective against vaginal transmission of HIV-1; since then, alternative TFV gel formulations have been explored as a prevention option for other high-risk groups. This includes rectal-specific formulations for individuals practicing condom- less receptive anal intercourse (CRAI), who have some of the highest rates of HIV acquisition. ${ }^{1}$ Ideally, rectal microbicides (RMs) will function similar to lubricants, which are already widely used by MSM or transgender women (TGW) practicing CRAI. ${ }^{2-4}$ They are therefore a promising alternative to oral TFV disoproxil fumarate/emtricitabine, which has recently been approved for use in preexposure prophylaxis (PrEP), but may be limited in efficacy by low levels of adherence among some PrEP users as demonstrated in the VOICE ${ }^{5}$ and FACTS $001^{6}$ trials.

\footnotetext{
${ }^{1}$ National HIV and Retrovirology Laboratory, JC Wilt Infectious Disease Research Centre, Public Health Agency of Canada, Winnipeg, Canada.

${ }^{2}$ Medical Microbiology and Infectious Diseases, University of Manitoba, Winnipeg, Canada.

${ }^{3}$ Fenway Health and Harvard Medical School, Boston, Massachusetts.

${ }^{4}$ Mass Spectrometry and Proteomics Core Facility, National Microbiology Laboratory, Public Health Agency of Canada, Winnipeg, Canada.

${ }^{5}$ HIV Center for Clinical and Behavioral Studies, Columbia University, New York, New York.

${ }^{6}$ University of Puerto Rico, San Juan, Puerto Rico.

${ }^{7}$ Department of Medicine, University of Pittsburgh, Pittsburgh, Pennsylvania.

${ }^{8}$ Department of Medicine Solna, Center for Molecular Medicine, Karolinska Institutet, Stockholm, Sweden.
}

(C) Laura Romas, et al., 2016; Published by Mary Ann Liebert, Inc. This Open Access article is distributed under the terms of the Creative Commons Attribution Noncommercial License (http://creativecommons.org/licenses/by-nc/4.0/) which permits any noncommercial use, distribution, and reproduction in any medium, provided the original author(s) and the source are credited. 
One of the key priorities in microbicide development is to exclude products that have the potential to cause mucosal injury. This might reduce product acceptability, and perhaps more importantly, diminish product efficacy. In the worst case scenario, a microbicide might increase the risk of HIV acquisition. The necessity for better evaluation of mucosal toxicity is exemplified by results of the early microbicide candidate Nonoxynol-9 (N-9), which although presumed nontoxic by prior safety assessments, nevertheless resulted in genital lesions, increased inflammation and epithelial toxicity, which were detrimental to its efficacy. ${ }^{7,8}$ Indeed, epithelial barrier disruption and inflammatory events within mucosal tissues have repeatedly been linked to poor protection against HIV transmission. ${ }^{9-11}$ Although a vaginal $1 \%$ TFV gel exhibited a good safety profile and minimal side effects in the CAPRISA-004 trial, ${ }^{12}$ data on rectal safety are limited. Preliminary evaluation of the vaginal formulation of $1 \%$ TFV gel used in the CAPRISA-004 trial for rectal use was associated with gastrointestinal intolerance (RMP-02/MTN-006), ${ }^{13}$ and adverse events (AEs) were attributed to a hyperosmolarity that was incompatible with the colorectal mucosal environment. ${ }^{14,15}$ A reduced glycerin vaginal formulation (RGVF) of a $1 \%$ TFV gel with lower osmolality has shown improved rectal safety and acceptability in the MTN-007 phase 1 study based on measurements of epithelial sloughing, fecal calprotectin, inflammatory cytokine levels, and cellular immune markers. ${ }^{16}$ However, these safety assessments may not capture all mucosal side effects. A systems biology analysis in the MTN-007 study associated TFV usage with mitochondrial dysfunction and altered cell proliferation pathways, ${ }^{17}$ which may indicate that existing assays for microbial toxicity are insufficient. Therefore, application of new technologies to evaluate the impact of rectal $1 \%$ TFV usage is warranted to provide a deeper insight into potential TFV or gel-mediated effects on rectal mucosa.

Mass spectrometry-based proteomics is a high-throughput and sensitive technique. ${ }^{18-20}$ Label-free methods are rapidly advancing in their ability to detect an increasing proportion of the proteome and have the added benefits of a reduced cost per sample relative to labeled methods, which make them ideal for analyzing large clinical cohorts. Indeed, their utility in assessing mucosal inflammation and other immune pathways makes them a useful addition to mucosal safety and toxicity monitoring studies. ${ }^{9,10,21}$ In this study, we evaluated this technique to assess mucosal proteome alterations of participants enrolled in the Project Gel: a phase 1 randomized, double-blind, multisite, placebo-controlled trial to assess the safety and acceptability of RGVF TFV gel in young men.

\section{Methods}

\section{Ethics}

The Project Gel study was approved by the Institutional Research Boards (IRB) at the University of Pittsburgh, the University of Puerto Rico, the New York State Psychiatric Institute, and the Fenway Health IRB. All participants signed an informed consent form before participating in the study.

\section{Study design}

Project Gel was completed in three stages, the first of which included baseline medical evaluations of 236 young MSM, 15 of which were sex workers (SW) and 8 were TGW (Stage 1A).
Participants who were diagnosed with anorectal infections (including chlamydia, gonorrhea, active HSV lesions, chancroid, genital ulcers, or genital warts), syphilis, Hepatitis B, or HIV infection were excluded from this study. Individuals who engaged in or planned to engage in CRAI with an HIV-infected partner were excluded from the trial. A subset of 124 MSM, including $10 \mathrm{SW}$ and $6 \mathrm{TGW}$ from Stage 1A, who met the aforementioned criteria and who reported at least one occasion of unprotected receptive anal intercourse (RAI) 3 months before enrollment were instructed to apply the universal hydroxyethyl cellulose (HEC) placebo gel before each CRAI act over a 3month period (Stage 1B). The first 24 eligible participants, who completed this phase with high adherence, were selected for a safety study, where they were randomized 1:1 to receive either $1 \%$ TFV or HEC placebo gel (Stage 2). The TFV gel used was modified from the RGVF with increased propylparaben content to improve gel stability. ${ }^{16}$ A baseline rectal sponge sample was taken 0-30 days before the first gel application (Visit 6, Dose 0). Participants returned to the clinic where a single dose of study gel was administered by a clinician, and a rectal sponge (\#405101; Merocel Sponges; Beaver Visitec) was taken by anoscopy $\sim 30 \mathrm{~min}$ after (Visit 7 , Dose 1). After a 1-week recovery period, participants, who did not experience AEs related to gel use, self-administered a once-daily outpatient dose for seven consecutive days and returned to the clinic for a final rectal sponge sampling (Visit 10, Dose 8). Sponges were stored at $-80^{\circ} \mathrm{C}$ until processing for mass spectrometry analysis.

\section{Mass spectrometry analysis of rectal sponge eluates using label-free proteomics}

Rectal mucosal samples $(n=72)$ from 24 participants across three collection points were processed and digested into peptides. Sponge samples were thawed, transferred to a 1.5-ml Eppendorf tube, and shaken in $300 \mu \mathrm{l} \mathrm{PBS}$ for $30 \mathrm{~min}$ at $4{ }^{\circ} \mathrm{C}$ and $500 \mathrm{rpm}$. Sponges and excess liquid were then transferred to a Costar Spin-X Centrifuge unit (Corning, Inc., Corning, NY) and spun at $10,000 \mathrm{~g}$ for $5 \mathrm{~min}$ at $4^{\circ} \mathrm{C}$. Rectal sponge eluate (RSE) was centrifuged again at 23,000 $\mathrm{g}$ for $10 \mathrm{~min}$ at $4^{\circ} \mathrm{C}$ to remove cellular debris. Supernatants were treated with $300 \mu \mathrm{l}$ of $8 \mathrm{M}$ urea-exchange buffer and stored at $-80^{\circ} \mathrm{C}$ until digestion. Treated RSE was digested in equal volumes using filter-aided sample preparation for label-free MS/MS as described previously. ${ }^{21}$ Samples were separated from salts and detergents using reverse-phase liquid chromatography (high pH RP, Agilent 1200 series micro-flow pump, Water XBridge column) using a step function gradient eluted into a single sample. Samples were quantified at the peptide level using a FluorProphile ${ }^{\circledR}$ Protein Quantification kit (SigmaAldrich, St. Louis, MO), and individual RSE samples (1 $\mu \mathrm{g}$ peptide per sample) were analyzed using an Easy nLC nanoflow LC system (Thermo Fisher Scientific, Waltham, MA) connected in line with an LTQ Orbitrap Velos mass spectrometer (Thermo Fisher Scientific) as described previously. ${ }^{21}$ Briefly, tryptic peptides were loaded onto a low-pH reversed-phase column $(0.075 \times 15 \mathrm{~cm})$, eluted over a 120 -min gradient $(2 \%-$ $30 \%$ acetonitrile, $0.1 \%$ formic acid), and nanosprayed into the LTQ Orbitrap Velos. The survey scans (MS1) were acquired in the Orbitrap at resolution of 60,000, and data-dependent acquisition was used to dynamically select the top 10 abundant peptide ions for fragmentation by CID. The peptide fragment ion scans (MS2) were acquired in the LTQ. 


\section{Analysis of mass spectrometry data}

Raw MS spectra were processed using Progenesis Software (v4.0; Nonlinear Dynamics, Durham, NC). Spectra were automatically aligned to a peptide mix control, with manual revisions for errors. Peptides with a charge state between +2 and +7 and a retention time between 10 and 125 min were retained. Filtered spectra were annotated using Mascot software (v2.4; Matrix Science, Boston, MA) using the UniProtKB/SwissProt (2012, v3.87) database for human and bacterial proteins. Searches were performed with the following criteria: carbamidomethyl (C) fixed modifications, oxidative $(\mathrm{M})$ variable modifications, a fragment ion mass tolerance of $0.5 \mathrm{Da}$, a parent ion tolerance of $10 \mathrm{ppm}$, tryptic enzyme digestion with a tolerance of one missed cleavage, and a decoy database. Search results were imported into Scaffold (v4.4.1.1; Proteome Software, Portland, OR) to filter protein identifications ( $80 \%$ confidence for peptide identification, $95 \%$ confidence for protein identification, and a minimum of two unique peptides identified per protein). Feature detection and quantification were all performed using default settings from the software.

\section{Statistical and multivariate analyses}

Protein abundance levels were normalized to total ion current (TIC) using Progenesis Software and exported for outlier filtration and further normalization. Outlier samples had a median normalized abundance greater than one standard deviation of the median normalized abundance of proteins identified across all samples. Protein identifications were limited to those with less than $25 \%$ technical variability. Retained samples ( $n=6 \mathrm{HEC}$ and $n=6 \mathrm{TFV}$ ) and protein identifications were subject to differential expression analysis. Protein levels from samples collected after 1 (V7) and 8 (V10) doses of either HEC or TFV gel were normalized to their respective baseline (V6), $\log _{2}$ transformed, and subsequently analyzed with paired and unpaired $t$-tests to determine changes in protein expression over time and between study arms, respectively $(p<.05$, two tailed, Benjimani-Hochberg corrected for multiple comparisons). Annotation of protein functions was completed using the functional enrichment software tool (FunRich) and the UniprotKB database. $^{22}$ Multivariate analysis was performed to identify predictive biomarkers of either HEC or TFV gel use as described previously. ${ }^{9,10}$ Briefly, the least absolute shrinkage and selection operator (LASSO) method was conducted with Matlab software (vR2015b; MathWorks, Natik, MA) to determine a minimum protein signature that best characterized individuals using either HEC or TFV gels [Dose 8 (V10) relative to baseline (Dose 0; V6) in each arm]. Partial least squares discriminant analysis (PLSDA) determined the predictive ability of LASSO factors to distinguish gel use in either arm relative to baseline samples. Differences in the frequency of RAI/CRAI and AEs between study arms were determined using Mann-Whitney $U$ tests $(\alpha=0.05)$. Grade 2 AEs associating with mild discomfort at the rectal mucosa were evaluated.

\section{Results}

\section{Participant characteristics}

Samples were collected from 24 young MSM across three study sites in the United States (Pittsburgh, PA; Boston, MA) and Puerto Rico (San Juan). Participants had a mean age of 23
Table 1. Sample Description at Baseline

\begin{tabular}{lllc}
\hline & $\begin{array}{c}H E C \\
(\mathrm{n}=12)\end{array}$ & $\begin{array}{c}T F V \\
(\mathrm{n}=12)\end{array}$ & $\begin{array}{c}\text { Total } \\
(\mathrm{n}=24)\end{array}$ \\
\hline $\begin{array}{l}\text { Demographic group } \\
N^{\mathrm{a}}(\%)\end{array}$ & & & \\
$\quad$ White & $5(42)$ & $5(42)$ & $10(42)$ \\
$\quad$ Latino/Hispanic & $4(33)$ & $5(42)$ & $9(38)$ \\
Other & $2(25)$ & $1(17)$ & $3(12)$ \\
Black & $1(8)$ & $1(8)$ & $2(8)$ \\
\hline
\end{tabular}

${ }^{\mathrm{a}}$ Total number of participants.

HEC, hydroxyethyl cellulose; TFV, tenofovir.

years, and were $42 \%$ White, 38\% Latino/Hispanic, $8 \%$ Black/ African American, and 12\% mixed/other race. Eight subjects from each site enrolled were randomized 1:1 to receive either a rectal-specific formulation of $1 \%$ TFV gel or HEC placebo gel. A summary of baseline demographics is presented in Table 1.

\section{Mass spectrometry analysis of rectal mucosal samples}

Samples were subject to strict outlier determination before downstream analysis. Box-and-whisker plots of protein abundance values in each of the 72 sample were created (Fig. 1A-C). Relative protein abundance levels, normalized to TIC [normalized abundance (NA)], were plotted to visualize outliers. Nonuniform distributions of total protein content were observed across samples at each collection point, as indicated by a large range of median NA levels at all collection periods. To limit experimental error related to variable protein levels, samples were restricted to one standard deviation from the average NA of all protein at each collection point (Supplementary Table S1; Supplementary Data are available online at www. liebertpub.com/aid). Samples with a median NA outside of 1 . $47 \times 10^{6}-6.47 \times 10^{6}$ (Dose 0), 4.88 $\times 10^{5}-6.12 \times 10^{6}$ (Dose 1), and $8.13 \times 10^{5}-7.21 \times 10^{6}$ (Dose 8 ) were determined to be outliers (red). A total of 17/72 samples across collection points were determined to be outliers, which corresponded to the elimination of six samples from either arm (Supplementary Table S1).

\section{Mucosal proteome changes associated with TFV gel usage}

Mass spectrometry analysis confidently identified 246 host (human) proteins across all HEC and TFV rectal mucosal samples after species and covariance filtration. Independent $t$-tests were performed to determine differences in rectal mucosal protein expression between TFV and HEC arms after one and eight doses. Volcano plots indicate that weak effects were observed between the HEC and TFV arms, with only $3 \%$ of proteins differentially abundant at either dosage (Fig. 2A, B); thus, differences in protein abundance could not be interpreted beyond random chance at $\alpha=0.05$. This was likely due to interpersonal variability across samples. Paired $t$-tests were also performed to look at intraindividual changes in rectal mucosal protein expression after either one or eight TFV/HEC doses relative to the baseline sample (Dose 0). Negligible changes in protein expression were observed in the HEC arm after Dose 1 (7 factors, or 3\%), while $17(7 \%)$ factors were significant at Dose 8 (Fig. 2C, D). A difference in the expression of several protein factors was observed after one application of TFV gel (16 proteins significant at $p<.05$ 
A

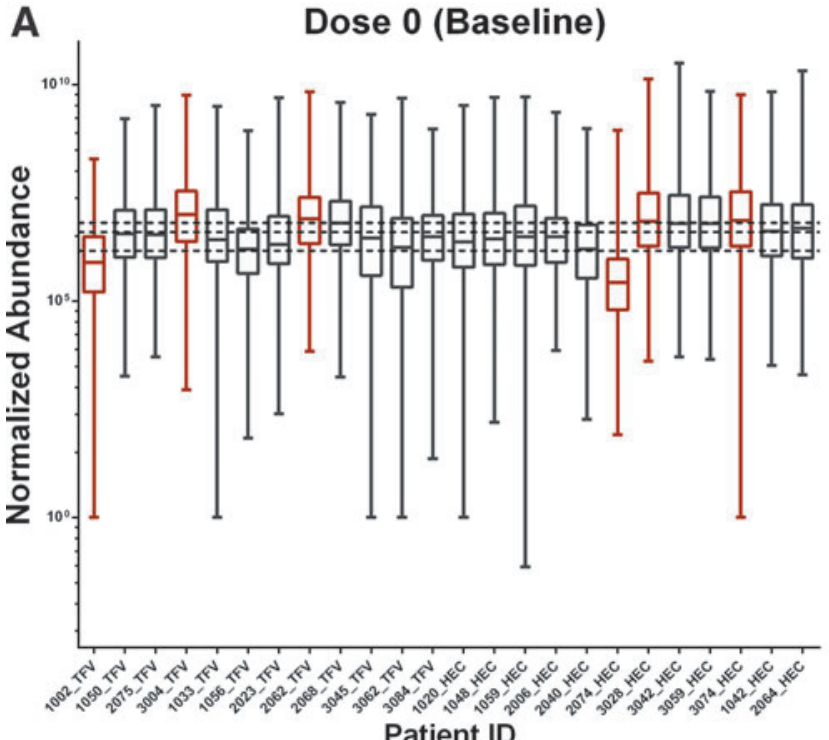

Patient ID

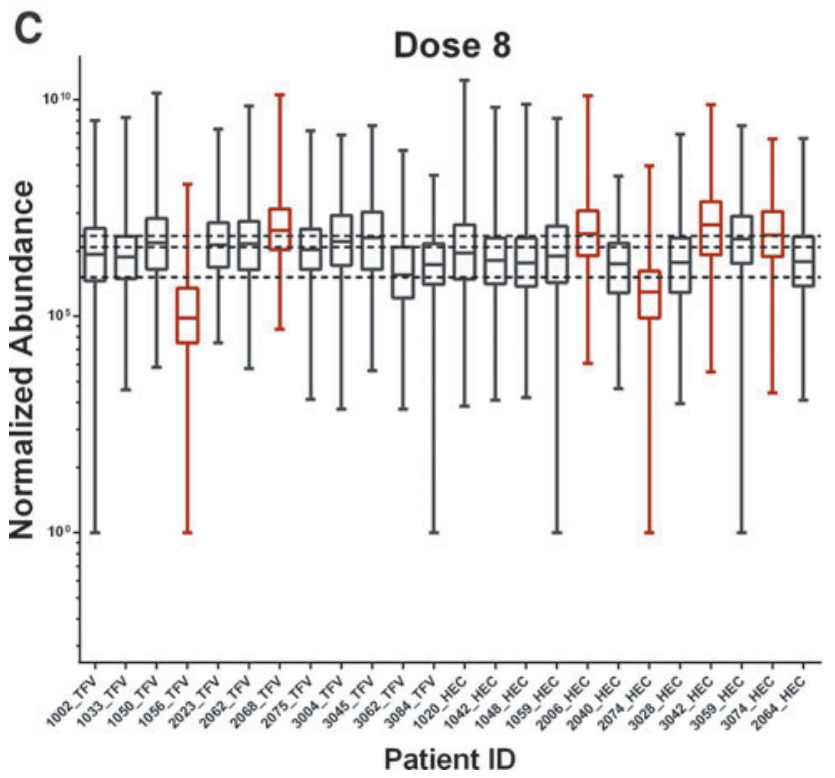

B

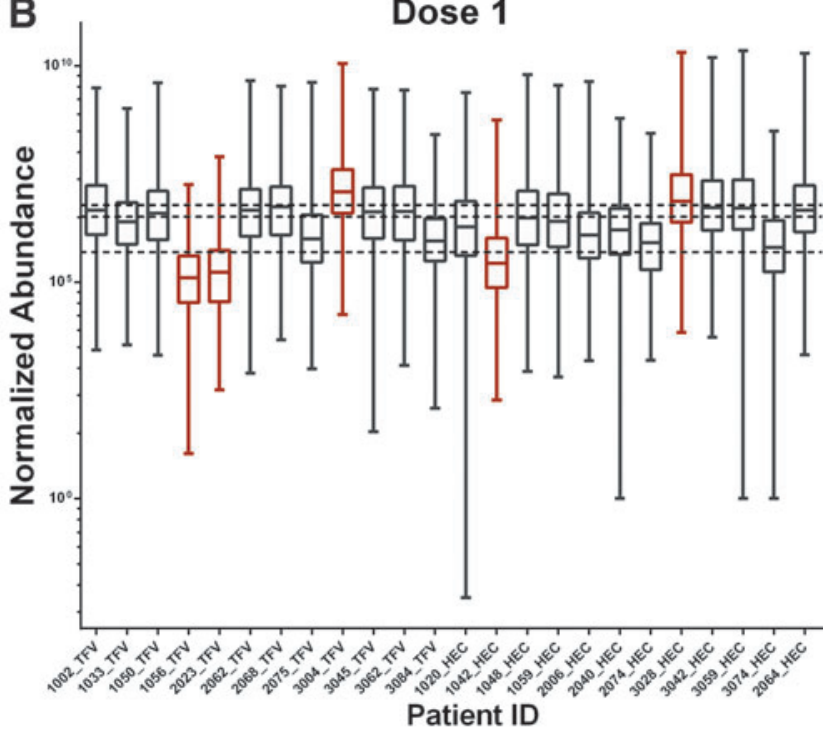

FIG. 1. Protein abundance distribution plots of rectal mucosal samples obtained by mass spectrometry. Box and whisker plots display the median, interquartile range, and minimum and maximum NA values for identified proteins in each sample taken at baseline (Dose 0; A), after one dose of either an HEC placebo or TFV rectal gel was administered (B), and after eight total doses of the same gel were applied (C). The average NA of each sample point was calculated across samples, and outlier samples were determined as having a median NA outside of a one standard deviation range from the average NA of all samples (dashed lines). Total protein content was found to be variable between samples at all three collection points (after Doses 0, 1, and 8). A total of 17 samples (6 samples collected at baseline, 5 from Dose 1, and 6 from Dose 8) were eliminated using this outlier criteria, which are highlighted in red in box-and-whisker plots. Participants who retained all three sample points ( $n=6 \mathrm{HEC}$ treated and $n=6 \mathrm{TFV}$ ) were retained for further analysis. HEC, hydroxyethyl cellulose; NA, normalized abundance; TFV, tenofovir. Color images available online at www.liebertpub.com/aid

and 1 protein significant at $p<.001)$; however, this was skewed toward underabundant proteins (Fig. 2E). The most prominent effects were observed after eight doses of TFV, which showed that $25(10 \%)$ proteins were significantly differentially expressed $(p<.05)$ with an equal distribution of proteins upregulated and downregulated (Fig. 2F), suggesting a potential alteration of biological pathways at the rectal mucosa. Trends observed after eight doses of TFV gel were confirmed using a local FDR calculation, which further supported the statistical significance of these changes (Sup- plementary Fig. S1). All statistical values obtained are located within the Supplementary Data.

\section{Proteome alterations with TFV gel usage associate with structural activity and epidermal development functions}

Functional enrichment analysis was performed on proteins significantly differentially expressed in the TFV arm $(p<.05)$ to identify any biological changes that may be 

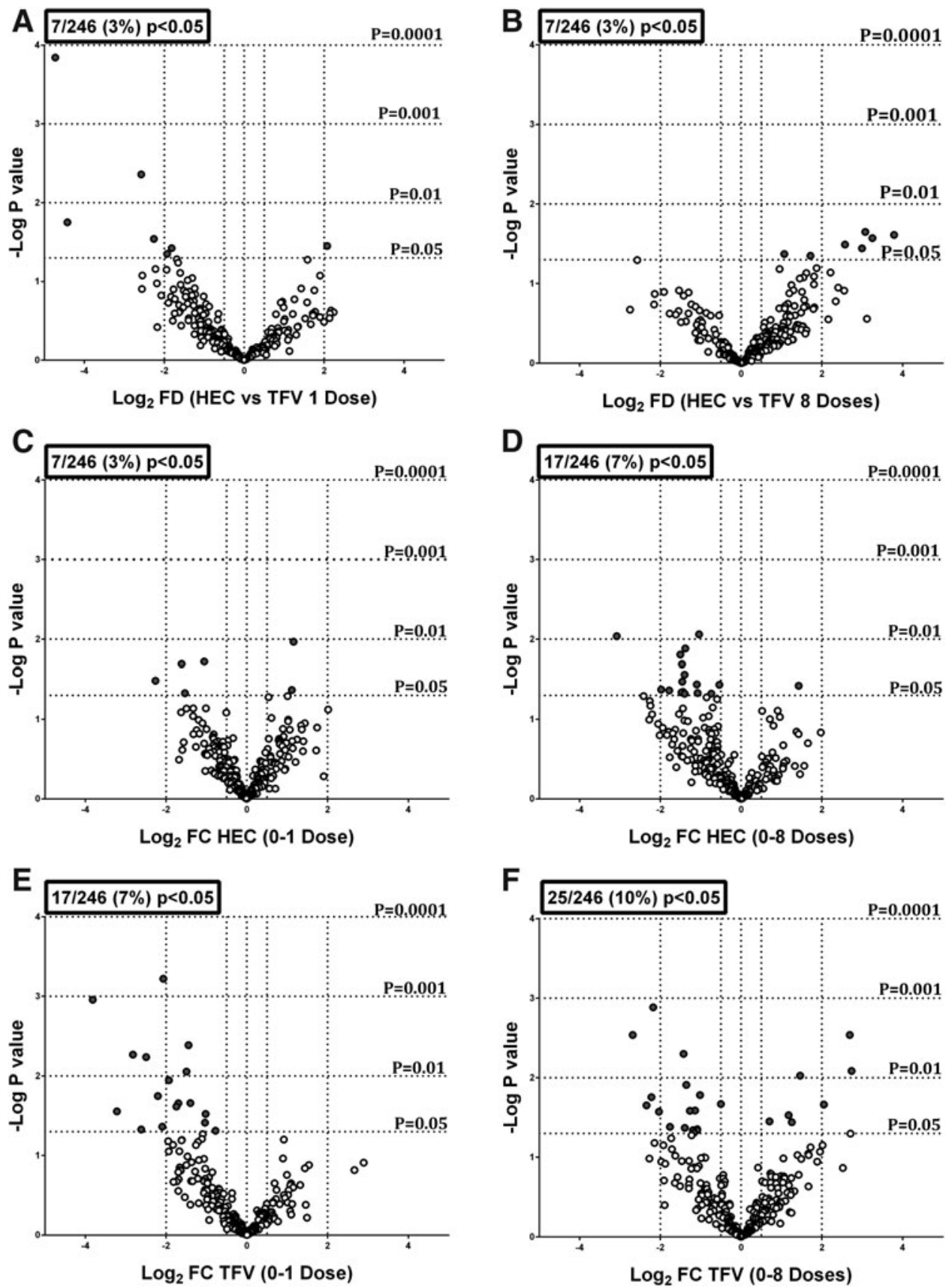

FIG. 2. Changes to the rectal mucosal proteome after daily applications of either $1 \%$ TFV or HEC placebo gel microbicide. Volcano plots display the $\log _{2 \text {-fold }}$ difference (FD) or $\log _{2 \text {-fold }}$ change (FC) values of protein expression along the $x$-axis and statistical significance values (-log $p$-value as determined by independent or paired $t$-tests) along the $y$-axis. Data points in gray indicate proteins significantly differentially expressed $(p<.05$; both paired and unpaired $t$-tests). A total of 246 unique proteins were identified in rectal mucosal samples after covariance and species filtering $(<25 \% \mathrm{CV}$ in reference sample, human only). Across-arm effects were evaluated using unpaired $t$-tests. Only $3 \%$ of proteins differentially expressed between HEC and TFV users at either time point (A, B). Paired $t$-tests of rectal protein levels after one or eight doses relative to a baseline sample were used to assess within-arm effects associated with either HEC or TFV gel usage. Less than $5 \%$ of proteins were differentially abundant after one dose of HEC (C), and only $7 \%$ changed after eight doses (D). Seven percent of proteins were differentially abundant after 1 TFV dose, with 1 factor significant at $p<.001$; however, the distribution of significant proteins was skewed toward underabundant factors (E). The strongest effect on rectal fluid protein expression associated with TFV gel use within individuals after eight doses, which showed $10 \%$ of proteins significantly differentially abundant, relative to baseline sample (F). 
associated with TFV use. A parallel analysis of factors significantly altered with HEC usage was performed to differentiate biological pathway alterations associated with TFV use from potential effects related to gel use in general. Enrichment analysis identified greater than 40 unique biological and molecular functions represented across study arms with the top 6 enriched functions in each arm displayed (Fig. 3). TFV-associated factors demonstrated enrichment in a diversity of molecular functions, including immune activity (peroxidases, heat shock proteins, antigen binding proteins), catalytic activity (lyases, cysteine/aspartic peptidases, transferase proteins), and structural components (cytoskeletal protein binding, structural molecules). Nine proteins significantly altered with TFV usage demonstrated a significant enrichment in structural proteins (adjusted $p=1.21 \times 10^{-10}$; KRT10, AFM, KRT1, KRT9, KRT6A, KRT13, TUBB4B, KRT5, $I V L)$ (Fig. 3A), which was not mirrored in the HEC study arm (adjusted $p=.31$ ). Furthermore, $12 \mathrm{TFV}$-associated factors were enriched for cell growth and/or maintenance functions (adjusted $p=3.1 \times 10^{-8} ;$ FN1, KRT10, AFM, FLNB, KRT1, KRT9, KRT6A, KRT13, TUBB4B, KRT19, KRT5, IVL) (Fig. 3B). Functional enrichment analysis was able to provide a global view of potential TFV-related effects on soluble factors within rectal secretions.

\section{Multivariate (LASSO) modeling identifies a signature of epidermal barrier proteins associated with TFV use at the rectal mucosa}

Multivariate modeling techniques were applied to the proteomic data to determine the minimum number of bio- markers needed to discriminate gel use in either the TFV or HEC arm based on covariance between proteins. The LASSO method has demonstrated increased sensitivity to detect significant protein changes between classes over alternative statistical methods ${ }^{23}$ and therefore is an appropriate addition to traditional univariate biomarker discovery. A minimum signature of 13 proteins was found to best distinguish the proteomic profile associated with 8 rectal applications of TFV (TFV Dose 8) relative to a baseline sample (TFV Dose $0)$. The model provided reliable classification of groups, with a calibration accuracy of $100 \%$ and $96 \%$ cross-validation accuracy. Latent variable 1 (LV1) was able to differentiate all baseline samples (negative scores on LV1) from those taken after repeated rectal TFV applications (positive scores on LV1), with minimal separation on LV2 (Fig. 4A). The majority of proteins (9/13) were negatively loaded on LV1, and were therefore positively associated with baseline protein expression and negatively associated with TFV, while four factors scored positively on LV1, indicating positive associations with TFV use (Fig. 4B). Interestingly, the model selected four factors that were identified as differentially abundant $(p<.05)$ in the univariate analysis $(F L N B, C A L M$, $C R N N, W D R 7)$, providing additional support for these factors as protein markers associated with TFV gel usage at the rectal mucosa (Fig. 4C). To determine if biomarkers were specific to TFV usage, parallel modeling experiments were performed on HEC rectal data. A total of 10 LASSO factors were identified, and PLSDA validation determined $100 \%$ calibration accuracy and $93 \%$ cross-validation accuracy. Two predictive HEC factors overlapped with the univariate analysis (AlAG1,K2C4); however, none of the TFV-
A

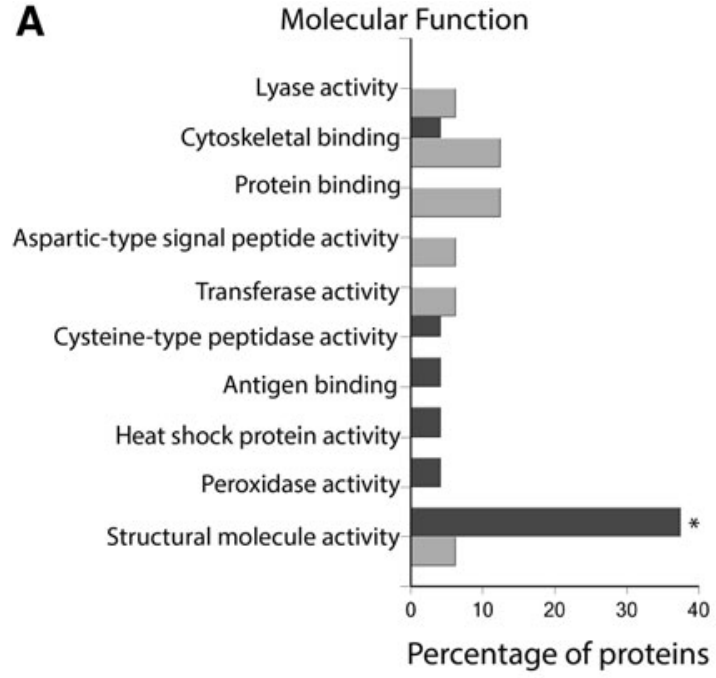

Tenofovir (TFV) Arm

Placebo (HEC)Arm

* Significant after Bonferroni correction

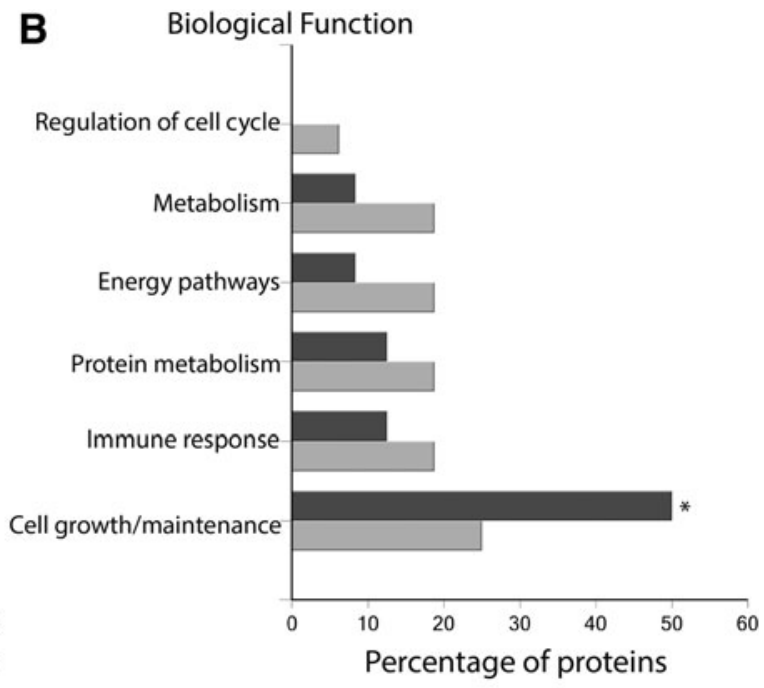

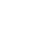



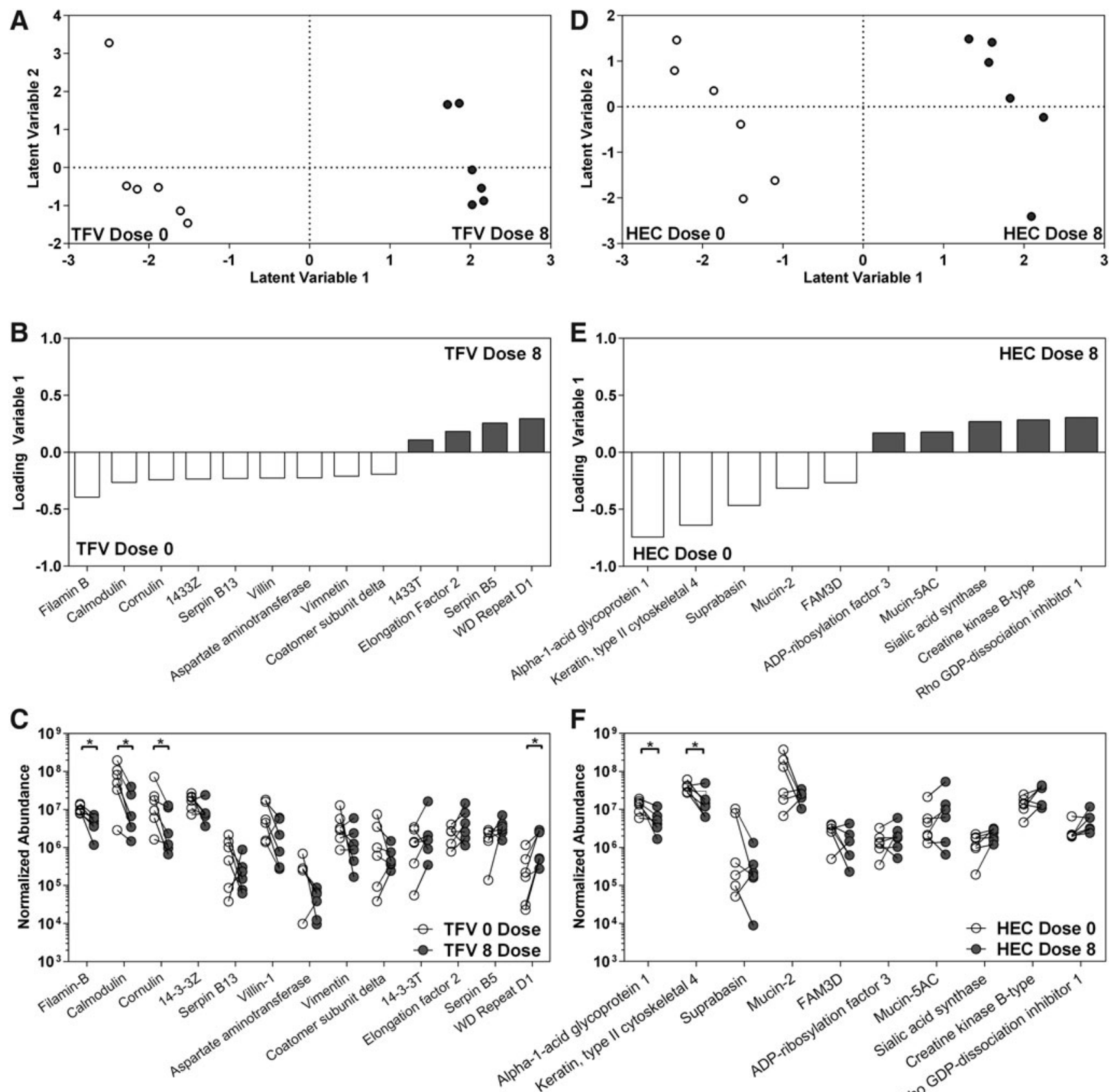

F

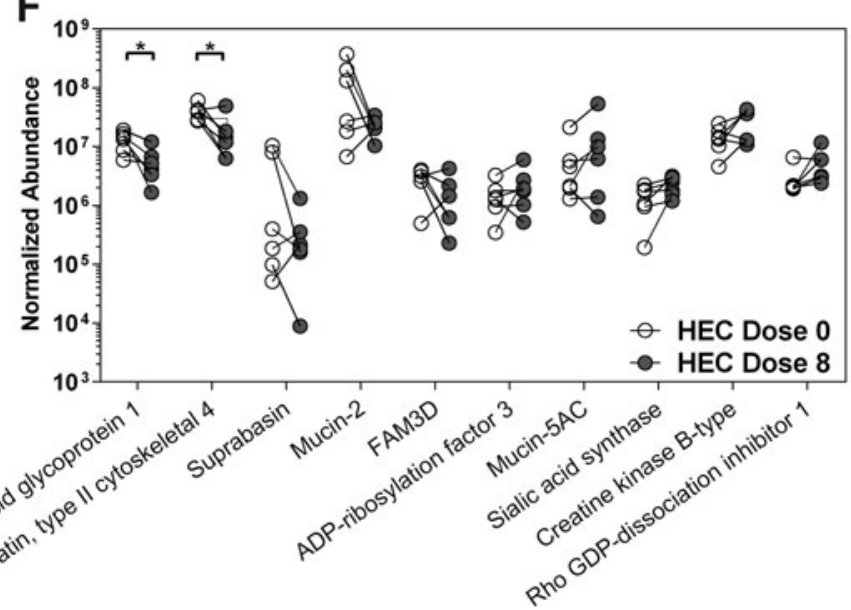

FIG. 4. A multivariate model accurately selects biomarkers of TFV-specific use within rectal mucosa, independent of HEC gel use. Multivariate modeling was performed using LASSO-selected biomarkers and PLSDA validation techniques to identify the minimal protein signature needed to classify repeated TFV usage within rectal mucosal fluid samples. LASSOselected mucosal proteins were able to predict TFV usage with 100\% calibration accuracy and 96\% cross-validation accuracy. The first two latent variables accounted for $46.1 \%$ of the total protein variance, with latent variable 1 showing accurate separation of baseline samples (white) from TFV samples (gray) (A). The scores plot demonstrates complete separation of samples collected during baseline (negative values on LV1) and those collected after eight daily TFV applications (positive values on LV1) based on the features selected by LASSO (B). The TFV-based LASSO model identified 13 biomarkers that could distinguish repeated TFV use from baseline. Four features positively associated with repeated TFV use, while nine were negatively associated. (C). Parallel modeling was performed on longitudinal HEC samples to determine if any TFV-associated factors could be attributed to general microbicide use. The HEC LASSO model demonstrated 100\% calibration and 93\% cross-validation accuracy with samples positively loaded on LV 1 associated with repeated HEC use (gray), and negatively loaded samples associated with a baseline sample (white) (D). Of the 10 LASSOselected features, 5 were negatively loaded, associating with the baseline sample, while the remaining 5 were associated with samples taken after 8 applications of HEC (E). Two LASSO factors identified in the HEC model overlapped with univariate analysis (F). PLSDA, partial least squares discriminant analysis. 
Table 2. Functional Annotation of LASSO Biomarkers Associated with TFV Use

\begin{tabular}{|c|c|c|c|c|}
\hline Accession & Description & $\begin{array}{l}\text { Mean L2FD } \\
\text { from baseline }\end{array}$ & $S D$ & Function \\
\hline CALM & Calmodulin & -2.68 & 1.21 & $\begin{array}{l}\text { Calcium ion signalling, tight junction assembly, cell } \\
\text { cycle progression }\end{array}$ \\
\hline CRNN & Cornulin & -2.22 & 1.56 & Epidermal differentiation protein, stress response, \\
\hline VILI & Villin-1 & -1.92 & 2.06 & $\begin{array}{l}\text { Cytoskeletal component, epidermal growth/ } \\
\text { differentiation factor, cell motility, regulation } \\
\text { of wound healing }\end{array}$ \\
\hline AATM & Aspartate aminotransferase & -1.91 & 2.75 & Amino acid metabolism \\
\hline VIME & Vimentin & -1.64 & 2.79 & Cytoskeletal component, apoptosis \\
\hline FLNB & Filamin-B & -1.36 & 0.87 & Cytoskeletal component, epithelial cell development \\
\hline SPB13 & Serpin B13 & -1.11 & 3.43 & $\begin{array}{l}\text { Serine and cysteine peptidase inhibitor, keratinocyte } \\
\text { development }\end{array}$ \\
\hline $1433 Z$ & 14-3-3 protein zeta/delta & -0.99 & 1.22 & $\begin{array}{l}\text { Adapter protein, cell signalling, apoptosis, blood } \\
\text { coagulation, Golgi assembly, membrane } \\
\text { organization, protein targeting, GTPase activity }\end{array}$ \\
\hline COPD & Coatomer subunit delta & -0.31 & 2.44 & Golgi-mediated transport \\
\hline EF2 & Elongation factor 2 & 0.86 & 1.34 & Transcription \\
\hline $1433 \mathrm{~T}$ & $14-3-3$ protein theta & 1.07 & 1.71 & $\begin{array}{l}\text { Adapter protein, cell signalling, apoptosis, blood } \\
\text { coagulation, Golgi assembly, membrane } \\
\text { organization, protein targeting, GTPase activity }\end{array}$ \\
\hline SPB5 & Serpin B5 & 1.24 & 1.96 & $\begin{array}{l}\text { Serine peptidase inhibitor, extracellular matrix } \\
\text { organization, tumor suppressor }\end{array}$ \\
\hline WDR1 & WD repeat-containing protein 1 & 2.73 & 1.58 & $\begin{array}{l}\text { Blood coagulation, platelet activation, cytoskeletal } \\
\text { component }\end{array}$ \\
\hline
\end{tabular}

associated factors were identified in the parallel HEC analysis (Fig. 4D-F).

LASSO biomarkers that varied within the TFV arm were largely cytoskeletal components or organization proteins (VILI, VIME, FLNB, SPB13, SPB5, WDR1), with some having additional roles in epidermal development $(C R N N$, $C A L M, F L N B, S P B 13, S P B 5$ ) (Table 2). Proteins identified in the HEC arm held functions in cellular metabolism (FAMD3, $A R F 3, K C R B, G D I R 1$, SIAS), while some factors held known functions in mucosal immunity (MUC2, MUC5AC, A1AG1); although most proteins and functions identified in the HEC arm were unique from TFV-associated biomarkers, one factor $(S B N)$ had an overlapping function in epidermal development (Table 3). Functional enrichment analysis suggests

Table 3. Functional Annotation of LASSO Biomarkers Associated with HeC Use

\begin{tabular}{|c|c|c|c|c|}
\hline Accession & Description & $\begin{array}{l}\text { Mean L2FD } \\
\text { from baseline }\end{array}$ & $S D$ & Function \\
\hline SBSN & Suprabasin & -1.71 & 2.89 & Epidermal differentiation \\
\hline $\mathrm{K} 2 \mathrm{C} 4$ & Keratin, type II cytoskeletal 4 & -1.50 & 1.02 & Cytoskeletal component, structural protein \\
\hline MUC2 & Mucin-2 & -1.45 & 2.51 & $\begin{array}{l}\text { Mucus component, cellular protein metabolism, } \\
\text { maintenance of gastrointestinal epithelium, } \\
\text { O-glycan processing }\end{array}$ \\
\hline A1AG1 & Alpha-1-acid glycoprotein 1 & -1.40 & 1.12 & $\begin{array}{l}\text { Acute phase inflammation, regulation of IL6, } \\
\text { regulation of TNF }\end{array}$ \\
\hline FAM3D & Protein FAM3D & -1.22 & 1.95 & $\begin{array}{l}\text { Response to dietary fats, regulation of insulin } \\
\text { secretion }\end{array}$ \\
\hline ARF3 & ADP-ribosylation factor 3 & 0.53 & 1.40 & $\begin{array}{l}\text { Protein transport, phospholipid metabolism, GTPase } \\
\text { activity, small molecule metabolism, } \\
\text { vesicle-mediated transport }\end{array}$ \\
\hline MUC5A & Mucin-5AC & 0.62 & 1.45 & $\begin{array}{l}\text { Mucus component, cellular protein metabolism, } \\
\text { maintenance of gastrointestinal epithelium, } \\
\text { O-glycan processing }\end{array}$ \\
\hline KCRB & Creatine kinase B-type & 0.80 & 1.05 & $\begin{array}{l}\text { Phosphate transfer, energy transfer, cellular chloride } \\
\text { homeostasis, nitrogen metabolism, creating } \\
\text { metabolic processes, small molecule metabolism }\end{array}$ \\
\hline GDIR1 & Rho GDP-dissociation inhibitor 1 & 0.91 & 1.00 & $\begin{array}{l}\text { Rho protein homeostasis, cellular response to redox } \\
\text { state, regulation of apoptosis, regulation of cell } \\
\text { adhesion, and migration, regulation of GTPase } \\
\text { activity }\end{array}$ \\
\hline SIAS & Sialic acid synthase & 0.96 & 1.14 & Cellular protein metabolism, lipid synthesis \\
\hline
\end{tabular}


Table 4. Frequency of Receptive Anal Intercourse and Reported Stage 2 Adverse Events

\begin{tabular}{lcrrr}
\hline & $H E C(\mathrm{n}=12)$ & $T F V(\mathrm{n}=12)$ & Total $(\mathrm{n}=24)$ & $\mathrm{p}$ \\
\hline $\begin{array}{l}\text { Sexual activity } \\
N^{\mathrm{a}} \text {, median (IQR, min-max) }\end{array}$ & $9,0(0-0,0-7)$ & $20,0(0-3,0-7)$ & & \\
$\quad R A I$ & $1,0(0-0,0-1)$ & $2,0(0-0,0-2)$ & $3,0(0-1,0-7)$ & .35 \\
$\quad C R A I$ & & & & .99 \\
Stage 2 AE & 0 & $3,0(0-0)$ & $2,0(0-0)$ & \\
$N^{\mathrm{b}}$, median (IQR) & $6,0(0-0.75)$ & $3,0(0-0)$ & $9,0(0-0)$ & .47 \\
$\quad$ Gel related & $12,2(0-2)$ & $17,1(0-2)$ & $29,1(0-2)$ & .65 \\
$\quad \begin{array}{l}\text { Procedure related } \\
\text { Unrelated }\end{array}$ & & & \\
\hline
\end{tabular}

${ }^{\mathrm{a}}$ Total number of sex acts.

${ }^{b}$ Total number of stage 2 AEs.

CRAI, condomless receptive anal intercourse; RAI, receptive anal intercourse.

that proteins that vary in expression with multiple TFV applications may be associated with changes in structure of the colorectal epidermis, beyond the changes what we would expect to see with microbicide use alone.

\section{Molecular signatures related to gel use were not confounded by sexual activity, and could not be associated with AEs}

As a signal associated with a loss of epidermal barrier integrity was observed with TFV gel use, we sought to determine if this disruption was due to an increase in receptive anal intercourse in the TFV arm, as mechanical abrasions or exposure to semen have the potential to drive our signature independent of gel use. The frequency of RAI or CRAI was compared across arms (Table 4). The total number of RAI events in the TFV arm were higher than those using the placebo gel; however, there was no significant difference in the median sex acts reported between groups $(p=.95)$. Only three instances of CRAI were reported during the trial, with no difference in the number of events between arms $(p=.99)$. Therefore, our evidence did not associate any prospective damage at the rectal mucosa with a skewed frequency of sexual activity across study arms.

Furthermore, to determine if this signature was related to clinical AEs, the frequency of reported grade 2 AEs was compared across study arms. There was no significant difference in the number of AEs relating to clinical procedures $(p=.65)$ or AEs that were unrelated to the study $(p=.36)$. No gel-related AEs were reported in the HEC arm; however, three events were reported across two individuals using the TFV gel. Although this subtle increase in clinical toxicity was observed with TFV gel use, it was not found to be significant (Table 4).

\section{Discussion}

This pilot analysis of samples from an RM phase I trial was performed to evaluate the effects of TFV gel usage on the host mucosal proteome, as well as develop the proteomic platform as a tool for mucosal toxicity monitoring within the context of a clinical trial. This study investigated mucosal proteome changes both longitudinally and cross-sectionally across study arms. In this analysis, we were able to identify greater than 250 proteins with more than 40 unique biofunctions represented in rectal sponge samples, highlighting the utility of this method to give an in-depth description of the colorectal mucosal environment. The largest changes to the proteome were observed after 8 daily rectal applications of $1 \%$ TFV gel in all comparisons. Multivariate modeling identified a protein signature that was consistent with TFV use, and proteins identified had known functions relating to the structural integrity of the epidermal barrier. These results suggest that exposure to TFV-based microbicides may impact epidermal function, an outcome which may have been undetected using conventional toxicity monitoring assays.

This analysis incorporated both univariate and multivariate analytical techniques to determine TFV-associated changes in rectal protein expression. Functional analysis showed an enrichment of structural molecules and cell growth/maintenance factors in the subset of significantly differentially expressed proteins after eight applications of TFV gel, which was independent of any factors associated with placebo gel use. The multivariate LASSO model identified 13 factors associated with TFV gel usage that could distinguish groups with $100 \%$ accuracy. Many of these biomarkers (filamin-B, cornulin, calmodulin, and WD-containing protein 1) were also identified in the univariate analysis. These proteins are components of the extracellular matrix of the mucosal epithelium and play important roles in tissue remodeling, epidermal growth and differentiation, cellular stress response pathways, and tight junction protein assembly. ${ }^{24-29}$ Several cytoskeletal components (villin-1 and vimentin) were also identified in this model, as well as serine antiproteases (serpin B13 and B5) that have been shown to regulate the breakdown of the extracellular matrix through protease inhibition. $^{30,31}$ We recently identified alterations of several of these proteins within mucosal secretions from women with high levels of proinflammatory cytokines in the female genital tract $^{9}$; thus, variance in these biological factors may suggest potential inflammatory effects of TFV gel upon the rectal epithelium. As this study identified an epidermal signature of interest, an important future direction may be to assess the degree of change in epithelial proteins relative to the time since last gel application, which may account for the rapid ${ }^{32}$ and dynamic $^{33-35}$ cell turnover rate of the colorectal epithelium. Furthermore, our findings add to the emerging evidence of side effects of TFV-gel usage, such as that from the MTN-007 trial, observing effects to mitochondrial function, epithelial cell proliferation, and alterations of cell differentiation pathways. ${ }^{17}$

It is important to note that although an investigation of the HEC placebo gel was not the focus of this study, mild 
changes in protein expression at the rectal mucosa were associated with its use. Recently, Smith-McCune et al. observed unexpected inflammatory events within the female genital tract of women using HEC gel, which were similar to effects observed in women using N-9 (a product known to damage mucosal epithelial tissue). ${ }^{36}$ Our LASSO model identified covariance of immune proteins (mucin-2 and alpha-1-acid glycoprotein 1), as well as variation in expression of the wound healing Rho ATPases (ADP-ribosylation factor 3 and Rho GDP-dissociation inhibitor 1) as predictors of HEC gel usage, ${ }^{37,38}$ and may suggest an association with altered mucosal immune pathways. Although the HEC gel has generally been considered safe for mucosal use, it has not been assessed in depth using omics platforms; therefore, our findings of subtle changes in immune protein levels with HEC gel use would be important to monitor in future clinical trials. However, in the absence of a positive control arm, such as nonoxynol-9, we are unable to assess how these alterations proteome relate to known sources of mucosal damage.

This study also provided useful information for the optimization of the proteomic platform in the context of a clinical trial. Our initial assessment of total protein content showed high variability across samples, many of which included baseline time points, which precluded those participants from being included in downstream analysis. This may be due to the nature of rectal mucosal samples, given the presence of fecal and other components from the GI tract, which may make normalization of total protein content before MS analysis challenging. Our variance subanalysis highlights the necessity of taking multiple samples at each study point, which is particularly important for those at baseline as elimination of the reference sample severely limits the interpretation of later collection points and reduced the overall power of this study. Additional wash-out steps of the colorectal lumen before sponge sampling may help alleviate this variability. Furthermore, independent high-powered assessments of the rectal fluid proteome and factors associating with its variability are needed to improve normalization techniques for these samples. The variability of rectal secretions notwithstanding, the analysis of mucosal fluid provides critical information on mucosal immune processes associated with HIV prevention therapy, with samples that are relatively noninvasive, making the approach an ideal addition to the repertoire of assays used to monitor mucosal safety in phase 1 clinical trials.

This study provides further evidence that TFV-based gels have effects on the rectal mucosal proteome. The changes in epidermal factors observed may reflect alterations to the primary mucosal barrier, which may have consequences for mucosal barrier functionality. Furthermore, it is unknown what long-term effects, if any, may be associated with these alterations. Future proteomic studies targeted at monitoring molecular changes that influence mucosal epithelial integrity may be warranted to fully determine the impact of TFV on mucosal safety.

\section{Acknowledgments}

We would like to thank all study participants and clinical staff from the study sites at the University of Pittsburgh, the University of Puerto Rico, and the Fenway Community Health Center. This work was supported through the National
Institutes of Health (R01 HD059533) (I.M., A.C.-D.), the Canadian Institutes of Health Research (OCB134115) (A.B.), and the Public Health Agency of Canada (A.B. and G.W.). Additional support came from the National Institute of Mental Health to the HIV Center for Clinical and Behavioral Studies at NY State Psychiatric Institute and Columbia University (P30-MH43520, PI Remien). L.R. holds a Canadian Institute of Health Research Master's Award.

\section{Author Disclosure Statement}

No competing financial interests exist.

\section{References}

1. Valleroy LA, MacKellar DA, Karon JM, et al.: HIV prevalence and associated risks in young men who have sex with men. Young Men's Survey Study Group. JAMA 2000; 284:198-204.

2. Giguere R, Dolezal C, Bauermeister JA, et al.: Influence of partner type on acceptability and likelihood of use of a rectal microbicide among young men who have sex with men in the United States and Puerto Rico. J Sex Res 2016; 53:633-641.

3. Pines HA, Gorbach PM, Reback CJ, Landovitz RJ, Mutchler MG, Mitsuyasu R: Commercial lubricant use among HIVnegative men who have sex with men in Los Angeles: Implications for the development of rectal microbicides for HIV prevention. AIDS Care 2014;26:1609-1618.

4. Peinado J, Lama JR, Galea JT, et al.: Acceptability of oral versus rectal HIV preexposure prophylaxis among men who have sex with men and transgender women in Peru. J Int Assoc Provid AIDS Care 2013;12:278-283.

5. Marrazzo JM, Ramjee G, Richardson BA, et al.: Tenofovirbased preexposure prophylaxis for HIV infection among African women. N Engl J Med 2015;372:509-518.

6. Rees H: FACTS 001 Phase III Trial of Pericoital Tenofovir $1 \%$ Gel for HIV Prevention in Women. Conference on Retroviruses and Opportunistic Infections. Seattle, WA, 2015.

7. Niruthisard S, Roddy RE, Chutivongse S: The effects of frequent nonoxynol-9 use on the vaginal and cervical mucosa. Sex Transm Dis 1991;18:176-179.

8. Tabet SR, Surawicz C, Horton S, et al.: Safety and toxicity of nonoxynol-9 gel as a rectal microbicide. Sex Transm Dis 1999;26:564-571.

9. Arnold KB, Burgener A, Birse K, et al.: Increased levels of inflammatory cytokines in the female reproductive tract are associated with altered expression of proteases, mucosal barrier proteins, and an influx of HIV-susceptible target cells. Mucosal Immunol 2016;9:194-205.

10. Birse K, Arnold KB, Novak RM, et al.: Molecular signatures of immune activation and epithelial barrier remodeling are enhanced during the luteal phase of the menstrual cycle: Implications for HIV susceptibility. J Virol 2015;89: 8793-8805.

11. Masson L, Passmore JA, Liebenberg LJ, et al.: Genital inflammation and the risk of HIV acquisition in women. Clin Infect Dis 2015;61:260-269.

12. Abdool Karim Q, Abdool Karim SS, Frohlich JA, et al:: Effectiveness and safety of tenofovir gel, an antiretroviral microbicide, for the prevention of HIV infection in women. Science 2010;329:1168-1174.

13. Anton PA, Cranston RD, Kashuba A, et al.: RMP-02/MTN006: A phase 1 rectal safety, acceptability, pharmacokinetic, and pharmacodynamic study of tenofovir $1 \%$ gel compared 
with oral tenofovir disoproxil fumarate. AIDS Res Hum Retroviruses 2012;28:1412-1421.

14. Rohan LC, Moncla BJ, Kunjara Na Ayudhya RP, et al.: In vitro and ex vivo testing of tenofovir shows it is effective as an HIV-1 microbicide. PLoS One 2010;5:e9310.

15. Fuchs EJ, Lee LA, Torbenson MS, et al:: Hyperosmolar sexual lubricant causes epithelial damage in the distal colon: Potential implication for HIV transmission. J Infect Dis 2007; 195:703-710.

16. McGowan I, Hoesley C, Cranston RD, et al.: A phase 1 randomized, double blind, placebo controlled rectal safety and acceptability study of tenofovir 1\% gel (MTN-007). PLoS One 2013;8:e60147.

17. Hladik F, Burgener A, Ballweber L, et al: : Mucosal effects of tenofovir 1\% gel. Elife 2015;4. DOI: 10.7554/elife.04525.

18. Bantscheff M, Kuster B: Quantitative mass spectrometry in proteomics. Anal Bioanal Chem 2012;404:937-938.

19. Bantscheff M, Lemeer S, Savitski MM, Kuster B: Quantitative mass spectrometry in proteomics: Critical review update from 2007 to the present. Anal Bioanal Chem 2012;404:939-965.

20. Zhu W, Smith JW, Huang CM: Mass spectrometry-based label-free quantitative proteomics. J Biomed Biotechnol 2010;2010:840518.

21. Romas LM, Hasselrot K, Aboud LG, et al:: A comparative proteomic analysis of the soluble immune factor environment of rectal and oral mucosa. PLoS One 2014;9:e100820.

22. Pathan M, Keerthikumar S, Ang CS, et al.: FunRich: An open access standalone functional enrichment and interaction network analysis tool. Proteomics 2015;15:25972601.

23. Tibshirani R: The lasso method for variable selection in the Cox model. Stat Med 1997;16:385-395.

24. Del Valle-Perez B, Martinez VG, Lacasa-Salavert C, et al:: Filamin B plays a key role in vascular endothelial growth factor-induced endothelial cell motility through its interaction with Rac-1 and Vav-2. J Biol Chem 2010;285: 10748-10760.

25. Salahshourifar I, Vincent-Chong VK, Chang HY, et al:: Downregulation of CRNN gene and genomic instability at $1 \mathrm{q} 21.3$ in oral squamous cell carcinoma. Clin Oral Investig 2015;19:2273-2283.

26. Kimchi ET, Posner MC, Park JO, et al.: Progression of Barrett's metaplasia to adenocarcinoma is associated with the suppression of the transcriptional programs of epidermal differentiation. Cancer Res 2005;65:3146-3154.

27. Kupfer DM, White VL, Jenkins MC, Burian D: Examining smoking-induced differential gene expression changes in buccal mucosa. BMC Med Genomics 2010;3:24.
28. Darragh J, Hunter M, Pohler E, et al.: The calcium-binding domain of the stress protein SEP53 is required for survival in response to deoxycholic acid-mediated injury. FEBS J 2006;273:1930-1947.

29. Shiomi R, Shigetomi K, Inai T, Sakai M, Ikenouchi J: CaMKII regulates the strength of the epithelial barrier. Sci Rep 2015;5:13262.

30. Gatto M, Iaccarino L, Ghirardello A, et al:: Serpins, immunity and autoimmunity: Old molecules, new functions. Clin Rev Allergy Immunol 2013;45:267-280.

31. Izuhara K, Ohta S, Kanaji S, Shiraishi H, Arima K: Recent progress in understanding the diversity of the human ovserpin/clade B serpin family. Cell Mol Life Sci 2008;65: 2541-2553.

32. Heath JP: Epithelial cell migration in the intestine. Cell Biol Int 1996;20:139-146.

33. Buchi KN, Moore JG, Hrushesky WJ, Sothern RB, Rubin $\mathrm{NH}$ : Circadian rhythm of cellular proliferation in the human rectal mucosa. Gastroenterology 1991;101:410-415.

34. Mariadason JM, Catto-Smith A, Gibson PR: Modulation of distal colonic epithelial barrier function by dietary fibre in normal rats. Gut 1999;44:394-399.

35. Shorter RG, Spencer RJ, Hallenbeck GA: Kinetic studies of the epithelial cells of the rectal mucosa in normal subjects and patients with ulcerative colitis. Gut 1966;7:593-596.

36. Smith-McCune K, Chen JC, Greenblatt RM, et al.: Unexpected inflammatory effects of intravaginal gels (Universal Placebo Gel and Nonoxynol-9) on the upper female reproductive tract: A randomized crossover study. PLoS One 2015;10:e0129769.

37. Leoni G, Neumann PA, Sumagin R, Denning TL, Nusrat A: Wound repair: Role of immune-epithelial interactions. Mucosal Immunol 2015;8:959-968.

38. Yamaguchi N, Mizutani T, Kawabata K, Haga H: Leader cells regulate collective cell migration via Rac activation in the downstream signaling of integrin beta1 and PI3K. Sci Rep 2015;5:7656.

Address correspondence to: Adam Burgener National HIV and Retrovirology Laboratory JC Wilt Infectious Disease Research Centre Public Health Agency of Canada 745 Logan Avenue Winnipeg, MN R3E $3 L 5$ Canada

E-mail: adam.burgener@umanitoba.ca 